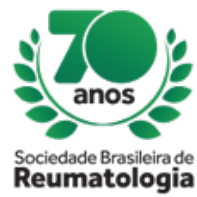

\title{
PYODERMA GANGRENOSUM AFTER REDUCTION MAMMOPLASTY
}

Bianca Alencar Dias Almeida (Hospital Universitário da Universidade Federal do Vale do São Francisco, PETROLINA, PE, Brasil), Rita Marina Soares de Castro Duarte (Hospital Universitário da Universidade

Federal do Vale do São Francisco, PETROLINA, PE, Brasil), Ernani Coelho Alencar (Hospital Universitário da Universidade Federal do Vale do São Francisco, PETROLINA, PE, Brasil), Edson Moreira Batista (Hospital Universitário da Universidade Federal do Vale do São Francisco, PETROLINA, PE, Brasil), Jandir Mendonça Nicacio (Hospital Universitário da Universidade Federal do Vale do São Francisco, PETROLINA, PE, Brasil), Leonardo Fernandes e Santana (Hospital Universitário da Universidade Federal do Vale do São Francisco, Petrolina, PE, Brasil), Sócrates Murilo Martins da Silva Oliveira (Hospital Universitário da Universidade Federal do Vale do São Francisco, PETROLINA, PE, Brasil)

\section{BACKGROUND}

Pyoderma gangrenosum (PG) is a neutrophilic dermatosis, which occurs in the absence of infection or primary vasculitis. They are usually manifested as painful papules, vesicles or nodules that progress with ulceration. It may appear spontaneously or be precipitated by trauma.

\section{CASE REPORT}

ASR, female, 33 years old. Previously healthy, without report of wound-healing deficits. Hospitalized due to fever, asthenia and vesicles in breasts eight days after reduction mammoplasty. The lesions coalesced and ulcerated with secretion of purulent content. There were extensive ulcerations, of regular contours and purulent base, painful, periareolar. The laboratory tests evidenced neutrophilia and increased inflammatory evidence. The biopsy was performed, which samples were sent to culture and histopathological analyses. Afterwards, blood cultures showed no evidence of bacterial growth. Despite the treatment, the patient maintained daily fever spikes. Due to the hypothesis of PG, pulse therapy with methylprednisolone $1 \mathrm{~g}$ was performed for three consecutive days. Patient progressed with improvement of pain and regression of lesions. She was discharged from hospital with oral prednisone associated with azathioprine. The glucocorticoid withdrawl was performed while other systemic causes as differential diagnosis were investigated. Antiphospholipid antibodies, anti-parietal cell antibody, anti-intrinsic factor were negative. Serum protein electrophoresis was normal. Antinuclear antibodies, anti-DNA and anti-Sm were negative, and the rheumatoid factor was non-reactive. Cryoglobulin screen, p-ANCA and c-ANCA were negative. The histopathology of skin biopsy revealed destructive neutrophilic inflammation. The culture of skin fragment showed no evidence of bacterial growth. The patient evolved with regression of the skin lesions after the maintenance therapy with oral prednisone and azathioprine.

\section{CONCLUSION}

PG is rare and its diagnosis depends on high clinical suspicion. Half of the cases have an association with other comorbidities, such as inflammatory bowel disease, rheumatological or hematological diseases or malignancy. The classic ulcer is extensive, has infiltrated edges, violaceous, necrotic background and granulation tissue. It is more common in lower limbs. The patergia, with trauma inducing or exacerbating the lesions, is reported. Typical histopathological findings include neutrophilic inflammation in the epidermis and dermis, vascular reaction with perivascular lymphocytic infiltrate, usually without vascular fibrinoid necrosis. Glucocorticoid is the basis of treatment. In disseminated or fast-onset disease and without any associated disease, pulse therapy can be performed. Azathioprine, mycophenolate mofetil and dapsone are options in refractory cases.

This case aims to alert the recognition of clinical presentation, triggering factors and differential diagnosis of $P G$, aiding in clinical reasoning and early treatment institution. 